\title{
Analysis of the Chemical Composition of Urinary Calculi using Fourier Transform Infrared Spectroscopy: A Preliminary Study
}

\author{
${ }^{1}$ Shalmoli Bhattacharyya, ${ }^{2}$ Gaurav Sharma, ${ }^{3}$ Arup Kumar Mandal, ${ }^{4}$ Shrawan Kumar Singh
}

\begin{abstract}
Background: The etiology of primary and recurrent stone formation in the urinary tract remains obscure in spite of intensive research. The formation of renal calculi is a multifactorial disorder resulting from the combined influence of epidemiological, biochemical and genetic risk factors which disturb the lithogenous salt profile in the body.

Materials and methods: In the present study, 52 stone samples have been analyzed for their stone composition by Fourier transform infrared spectroscopy (FTIR). The samples were made in a potassium bromide pellet and analyzed to identify the stone components.
\end{abstract}

Results: Stone formation was more prevalent in males and the incidence increased with age. Majority of stones were oxalate and mixed stones. The components of mixed stones were identified by spectrum analysis.

Conclusion: Specific dietary factors can affect the urinary composition and supersaturation, which in turn, can affect the process of crystallization and stone formation. The same chemical components may crystallize in different forms during stone formation. Hence, proper analysis has to identify not only the molecular species present in the calculus, but also the crystalline form. So, quantitative evaluation of all components of the calculi is necessary for effective prevention or reduction of stone recurrences.

Keywords: Urinary calculi, Fourier transform inframed spectroscopy, Spectrum.

How to cite this article: Bhattacharyya S, Sharma G, Mandal AK, Singh SK. Analysis of the Chemical Composition of Urinary Calculi using Fourier Transform Infrared Spectroscopy: A Preliminary Study. J Postgrad Med Edu Res 2014;48(3):128-131.

Source of support: Nil

Conflict of interest: None

\section{INTRODUCTION}

Urinary stone disease causes substantial discomfort, pain and occasional renal failure. ${ }^{1}$ Urinary stones are hard solid

\footnotetext{
${ }^{1}$ Assistant Professor, ${ }^{2}$ Senior Research Fellow, ${ }^{3,4}$ Professor

1,2Department of Biophysics, Postgraduate Institute of Medical Education and Research, Chandigarh, India

${ }^{3,4}$ Department of Urology, Postgraduate Institute of Medical Education and Research, Chandigarh, India
}

Corresponding Author: Shalmoli Bhattacharyya, Assistant Professor, Department of Biophysics, Postgraduate Institute of Medical Education and Research, Chandigarh, India, Phone: 01722755251, e-mail: shalmoli2007@yahoo.co.in pellets, and are found in the urinary tract (kidney, ureter and bladder). Studies have demonstrated that genetic, geographical, climatic and seasonal factors have a role in the process of lithogenesis. In India, high incidence of calculi has been reported especially in Gujarat, Rajasthan, Punjab and Madhya Pradesh. ${ }^{2}$ According to a report by Sinha et al, ${ }^{3}$ $12 \%$ of the Indian population is expected to have urinary stones, out of which upto $50 \%$ may suffer kidney damage. Factors contributing to increased risk of renal stones include family history, some disease conditions like Crohn's disease, urinary tract infections, renal tubular acidosis, hyperparathyroidism, medullary sponge kidney, and Dent's disease. ${ }^{4}$ Marked regional variations have been observed in the stone prevalence that has led to the recognition of stone belts. It also has been suggested that water fluoridation, the addition of fluoride to drinking water, is responsible for some cases of kidney stones. Anatomical abnormalities of the urinary tract and certain drugs like aspirin, opiramate (Topamax), a drug commonly prescribed to treat seizures and migraine headaches, some diuretics, vitamin D supplements, calciumcontaining antacids, and the protease inhibitor indinavir (Crixivan) a drug used to treat HIV infection and recurrent infections may be associated with increased risk of kidney disease. ${ }^{5,6}$ Various therapeutic strategies including diet management, diuretics, expulsion therapy, chelating agents and probiotic therapy have been applied in combinations to have effective treatment. ${ }^{7}$ Dietary intervention based on chemical composition and health education, in this regard, may be helpful as a preventive measure. Information about the stone composition provides an indication of the abnormal urinary milleu and guides comprehensive metabolic evaluation to identify underlying risk factors.

Urinary stones can be classified based on a number of factors like size, shape, location and composition (mineralogy). Reliable analytical information is very important to study the etiology of stone formation. Broadly, stones are classified into four types, according to their chemical composition, e.g. calcium stone (calcium oxalate and calcium phosphate), uric acid stone, magnesium ammonium phosphate (struvite) stone, cystine stone. ${ }^{8}$ The highly variable composition of urinary calculi has led to the development of different methods of stone analysis. Infrared (IR) spectroscopy, using Fourier transformation is a specific, rapid, and 
versatile analytical technique which provides qualitative as well as a semiquantitative analysis of kidney stone composition. Furthermore, using Fourier transform infrared spectroscopy (FTIR), the quantification of the relative amount of each constituent is possible without using any solvent. The analysis is based on the principle that every molecule gives a characteristic absorption spectrum in the IR region depending on the chemical bonding conditions and also on the particular structure. The vibration motions of atoms in covalent bonds are measured by passing IR radiation through the powdered stone sample, which has been compressed into a nearly transparent wafer with adequate quantity of potassium bromide. Oscillations induced in the FTIR region $\left(4000-400 \mathrm{~cm}^{-1}\right)$ in molecules and crystals, bring about an alteration of the dipole moment of the oscillating system as characterized by its signature spectrum scan.

We have undertaken a pilot study, on the analysis of constituents of urinary stones, from a subset of patients to get a preliminary data on the major stone constituents prevalent in this region. Knowledge of chemical composition of renal stones may be of great importance, both as a guide for planning the policy of medical management, and also for better understanding of physicochemical principles underlying the formation of calculi. This may help to give advice and suggestions for patients to initiate preventive measures in reducing the risk of prevalence and recurrence of urolithiasis.

\section{MATERIALS AND METHODS}

A total of 52 urinary stone samples for the present study were referred from the department of Urology of our institute for routine analysis by FTIR in our laboratory. All stones were washed with distilled water and dried completely in an incubator. The sample was grounded with a pestle and mortar until a fine homogeneous powder was obtained. For the FTIR spectroscopic investigation, the powdered urinary stone material was homogeneously mixed with potassium bromide $(\mathrm{KBr})$, an inert carrier, which did not show any absorption in the spectral region to be investigated. It was then pressed into a pellet using evacuated disk under high pressure in a hydraulic pellet press (Specac, USA). The pellet with $\mathrm{KBr}$ was inserted into the IR sample holder. The spectroscopy was performed using spectrum two FTIR spectrometer (Perkin Elmer, UK) and scan profile was obtained from an average of 50 scans/sample. The data was analyzed using spectral database (ST-Japan-Europe GmbH).

\section{RESULTS AND DISCUSSION}

Gender-wise distribution of renal stones in our study group revealed a higher incidence in males $(73 \%)$ as compared to females $(27 \%)$ with male to female's ratio of $2.7: 1$ (Table 1). Increased incidence of urinary calculi in males has been attributed to increased dietary protein intake, which increases urinary excretion of phosphates and magnesium and reduces urinary citrate concentration while the lower risk of stone formation in women was attributed initially to increased urinary citrate concentrations due to the lower urinary saturation of stone forming salts. ${ }^{9}$ However, subsequent experiments in animals demonstrated that testosterone promoted crystal growth by suppressing osteopontin expression in the kidney and increasing urinary oxalate excretion while estrogen possibly inhibited stone formation by increasing osteopontin expression in the kidney and decreasing urinary oxalate excretion. ${ }^{10-12}$ Age-wise distribution in Table 2 revealed that stone formation was higher in the older population (above 30 years). This may be attributed to the fact that older stone formers have been reported to excrete less urinary calcium than their younger counterparts ${ }^{13}$ and may exhibit defects in urinary inhibitors of crystallization. ${ }^{14}$

When IR radiation is incident on the urinary stone, characteristic attenuations of the incident energy occur, depending on the ability of the molecules, in the compound to oscillate at certain wavelengths. The number of oscillations possible in a molecule depends on the number and nature of the atoms present and the angular structure of the molecule. ${ }^{15}$ In the present study, all FTIR spectra were identified with matching percentages of IR spectra of the reference compounds. A total of 52 stones were analyzed for the composition (Table 3) and it was found that most of the stones analyzed were of calcium oxalate (weddelite and whewellite). Calcium oxalate urinary stones occurred more frequently in males than in females. Supersaturation, crystal nucleation, and crystal adherence to the surface of renal epithelial cells are considered initiating events in the genesis of calcium oxalate monohydrate stones. ${ }^{16,17}$ There is strong evidence that tubular dysfunction or damage is involved in calcium oxalate monohydrate stones binding and subsequent pathology. ${ }^{18,19}$ Oxalate is an end product of several metabolic pathways (including those involved in serine, glycine, hydroproline and ascorbate). A total of 10 to $20 \%$ urinary oxalate is derived from dietary sources and food rich in oxalate are cranberries, spinach, chocolate and tea. ${ }^{20,21}$ Oxalates were differentiated by a strong band

Table 1: Patient distribution according to gender

\begin{tabular}{lll}
\hline Sex & Number of patients & Percentage \\
\hline Male & 38 & 73 \\
Female & 14 & 27 \\
\hline
\end{tabular}

Table 2: Percent distribution of patients according to age

\begin{tabular}{lll}
\hline Age & Number & Percentage \\
\hline $0-18$ & 6 & 11.5 \\
$18-30$ & 9 & 17.3 \\
Above 30 & 37 & 71.1 \\
\hline
\end{tabular}


Table 3: Stone composition of the patients

\begin{tabular}{lll}
\hline Major stone composition & Number of stones & Percentage \\
\hline $\begin{array}{l}\text { Mixed stones } \\
\text { Calcium oxalate }\end{array}$ & 20 & 38.4 \\
(Mono + di-hydrate) & 22 & 42.3 \\
Calcium oxalate monohydrate & 8 & 15.3 \\
$\begin{array}{l}\text { Carbonate apatite } \\
\text { Amorphous calcium }\end{array}$ & 1 & 2 \\
phosphate carbonate & 1 & 2 \\
\hline
\end{tabular}

$(\mathrm{C}=\mathrm{O})$ at $1620 \mathrm{~cm}^{-1}$ asymmetric to the left, a modest band $(\mathrm{C}-\mathrm{O})$ near $1316 \mathrm{~cm}^{-1}$, and a valence vibration of $\mathrm{H}_{2} \mathrm{O}$ in staircase form at 3500 to $2700 \mathrm{~cm}^{-1}$ with maxima at $3490 \mathrm{~cm}^{-1}$ as observed in Figure 1. The distinctive out of the plane deformation peaks were found in the fingerprint region, at $781 \mathrm{~cm}^{-1}$, and secondary vibrations at 517,885 and $951 \mathrm{~cm}^{-1}$. The absorption band at $885 \mathrm{~cm}^{-1}$ is due to $\mathrm{C}=\mathrm{C}$ stretching mode. The bands at 661 and $781 \mathrm{~cm}^{-1}$ are due to the out-of-plane $\mathrm{O}=\mathrm{H}$ bending and $\mathrm{C}=\mathrm{H}$ bending mode, respectively and band at $517 \mathrm{~cm}^{-1}$ arises due to $\mathrm{O}=\mathrm{C}=\mathrm{O}$ in-plane bending as shown by Sundaramoorthi and Kalainathan. ${ }^{22}$

In our study, calcium oxalates, both monohydrate (whewellite) and dihydrate (weddellite) together were found in 22 samples while another 8 samples consisted of calcium monohydrate only. Carbonate apatite alone was found in one sample. Only one stone sample was found to contain amorphous calcium phosphate carbonate. Excess calcium is normally removed from the blood by kidneys and excreted in the urine. In hypercalciuria, excess calcium builds up in the kidneys and urine, where it combines with other waste products to form stones. Low levels of citrate, high levels of oxalate and uric acid, and inadequate urinary volume may also cause calcium stone formation. Calcium phosphate stones typically occur in patients with metabolic or hormonal disorders, such as hyperparathyroidism and renal tubular acidosis. ${ }^{23}$

A major fraction of the analyzed stones were composed of mixed compounds. There were three stones having magnesium ammonium phosphate (struvite) as the major component, six stone samples showed the presence of uric acid as the major constituent on spectra analysis. Carbonate apatite (10 samples) and ammonium urate (4 samples) were the other minerals observed in the mixed stones. In the sample containing struvite, the characteristic IR spectrum showed a strong band at $1008 \mathrm{~cm}^{-1}$ due to the absorption of the phosphate group. Phosphate groups were reported to show an absorption range of $1000-1100 \mathrm{~cm}^{-1} \cdot{ }^{24}$ Presence of other bands were observed at 1435,761, and $572 \mathrm{~cm}^{-1}$. The band at $1435 \mathrm{~cm}^{-1}$, due to vibration of $\mathrm{NH}_{4}$ group, was observed in the spectrum (Fig. 2). When struvite was in association with apatite, the presence of latter was deduced by absorption at $600 \mathrm{~cm}^{-1}$ and by a shift of the band at $1008 \mathrm{~cm}^{-1}$ towards higher frequencies. Uric acid had strong peaks at $619,705,1025 \mathrm{~cm}^{-1}$ that corresponded to $\mathrm{CH}_{2} \mathrm{OH}$ groups and the $\mathrm{C}=\mathrm{O}$ stretching vibration coupled with $\mathrm{C}-\mathrm{O}$ bending of the $\mathrm{C}=\mathrm{OH}$ groups of carbohydrates. The band at $1122 \mathrm{~cm}^{-1}$ corresponded to $\mathrm{C}=\mathrm{O}$ vibration of carbohydrates. Bands at 1307 and $1348 \mathrm{~cm}^{-1}$ were due to amide III, $\mathrm{C}=\mathrm{O}$ stretching vibrations and $\mathrm{N}=\mathrm{H}$ in plane bending, $1435 \mathrm{~cm}^{-1}$ due to in plane bending $\left(\mathrm{CH}_{2}\right), 1589$ $\mathrm{cm}^{-1}$ due to ring $\mathrm{C}=\mathrm{C}$ stretch of phenyl and at $3015 \mathrm{~cm}^{-1}$ due to vibration in $\mathrm{CH}$ of lipids (Fig. 3). In our study, uric acid was found to be a major constituent in about $11.5 \%$ of the total stones. Genetics and metabolic disorder may play a role in the development of uric acid stones, which are more common in men. Struvite and carbonate stones were relatively less prevalent. Struvite is also called an infection stone, since magnesium ammonium phosphate is formed during infection with urease producing bacteria which affects the chemical balance of the urine. ${ }^{25}$

The occurrence of renal stone disease is related to food habits of individuals. Dietary factors include a high intake of animal proteins and oxalates and a low intake of potassium containing citrus fruits and fluids. ${ }^{26}$ Inadequate fluid consumption decreases total urinary volume thereby increasing the concentration of stone forming salts. Intake of sodium is also associated with increased risk of stone formation

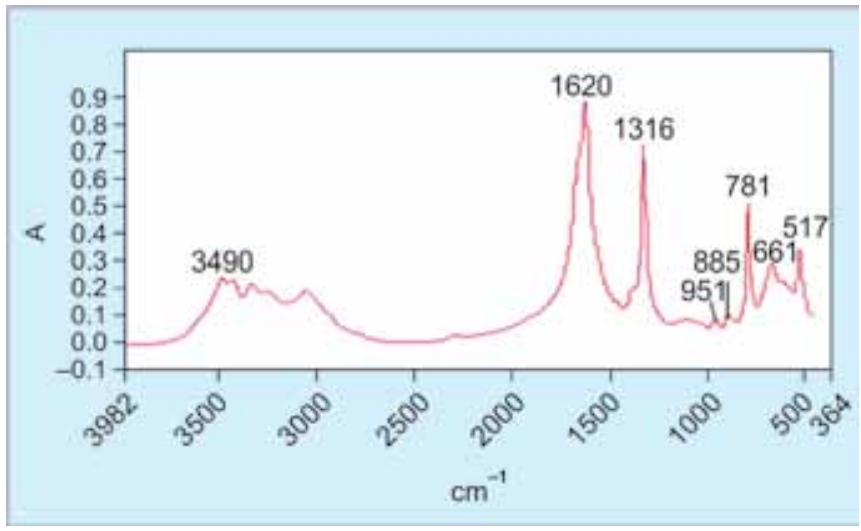

Fig. 1: FTIR-spectrum profile of a calcium oxalate stone showing major peaks

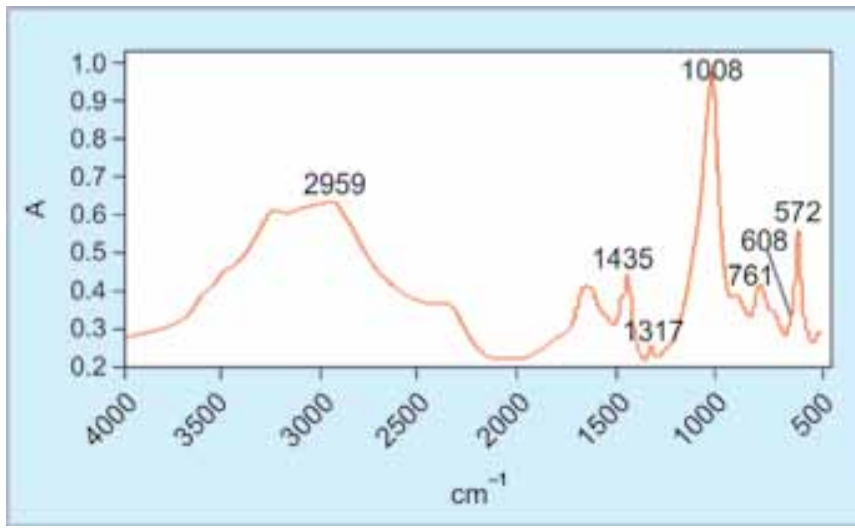

Fig. 2: FTIR-spectrum profile of a mixed stone containing $80 \%$ magnesium ammonium phosphate (struvite) and $20 \%$ amorphous calcium phosphate carbonate 


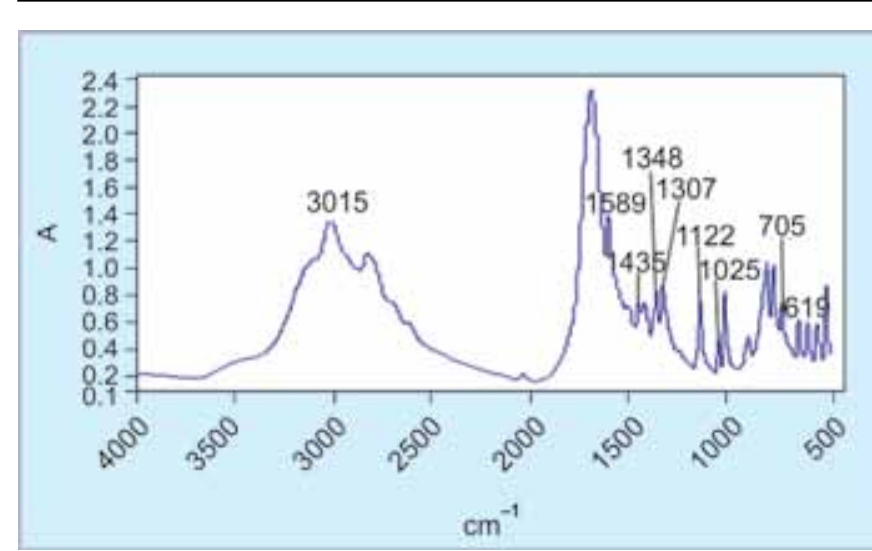

Fig. 3: FTIR-spectrum profile of a mixed stone with high uric acid content $(80 \%)$

presumably, because of increased urinary calcium excretion. ${ }^{27}$ Thus, for rational, efficient, and specific urolithiasis management, further studies in a larger population is needed to compare and contrast the choice of using FTIR for the characterization of crystalline and noncrystalline stone samples. Composition of the calculi provides information that could be useful for finding out the underlying cause of kidney stone formation, especially in recurrent stone disease. It provides an option to decide whether to treat the patients therapeutically or surgically. A study of the chemical composition of renal stones is important for understanding their etiology as well, planning proper management of the disease and prevent its recurrence. Proper dietary modifications guided by the stone composition may considerably decrease the morbidity and cost associated with recurrent stone disease. Extensive area-wise studies need to be carried out to assess the real picture of stone burden, its composition and its correlation with environmental, dietary and metabolic state of the patient.

\section{ACKNOWLEDGMENT}

The authors acknowledge the technical help rendered by Mr Kuldeep.

\section{REFERENCES}

1. Bhatt PA, Paul P. Analysis of urinary stone constituents using powder X-ray diffraction and FTIR. J Chem Sci 2008;120(2): 267-273.

2. Vyas AS, Patel MB, Patel AI, Joshi NR. Epidemiology of renal stone ailment in few districts of Gujarat state. Pharma Science Monitor 2012;3(2):232-239.

3. Sinha T, Karan SC, Kotwal A. Increased urinary uric acid excretion: a finding in Indian stone formers. Urol Res 2010; 38(1):17-20.

4. Worcester EM, Coe FL. Nephrolithiasis. Prim Care 2008;35(2): 369-391.

5. Jackson RD, LaCroix AZ, Gass M, Wallace RB, Robbins J, Lewis CE, Bassford T, Beresford SA, Black HR, Blanchette P, et al. Calcium plus vitamin D supplementation and the risk of fractures. N Engl J Med 2006;354(7):669-683.

6. Chan-Tack KM, Truffa MM, Struble KA, Birnkrant DB. Atazanavir-associated nephrolithiasis: cases from the US food and drug administration's adverse event reporting system. AIDS 2007;21(9):1215-1218.
7. Bijarnia RK, Kaur T, Singla SK, Tandon C. A novel calcium oxalate crystal growth inhibitory protein from the seeds of Dolichos biflorus (L). Protein J 2009;28(3-4):161-168.

8. Garrow JS, James WPT, Ralph A. Human nutrition and dietetics. New York: Churchill Livingstone 2000; p 37-59.

9. Welshman SG, McGeown MG. The relationship of the urinary cations, calcium, magnesium, sodium and potassium in patients with renal calculi. Br J Urol 1975;47(3):237-242.

10. Yagisawa T, Ito F, Osaka Y, Amano H, Kobayshi C, Toma H. The influence of sex hormones on renal osteopontin expression and urinary constituents in experimental urolithiasis. J Urol 2001; 166(3):1078-1082.

11. Parmar MS. Kidney stones. BMJ 2004;328(7453):1420-1424.

12. Abbagani S, Gundimeda SD, Varre S, Ponnala D, Mundluru HP. Kidney stone disease: etiology and evaluation. IJABPT 2010; 1(1):175-182.

13. Goldfarb DS, Parks JH, Coe FL. Renal stone disease in older adults. Clin Geriatr Med 1998;14(2):367-381.

14. Bergsland KJ, Kinder JM, Asplin JR, Coe BJ, Coe FL. Influence of gender and age on calcium oxalate crystal growth inhibition by urine from relatives of stone forming patients. J Urol 2002; 167(6):2372-2376.

15. Kanchana P Sundaramoorthi, Jeyanthi GP. Biochemical analysis and FTIR-spectral studies of artificially removed renal stone mineral constituents. J Minerals and Materials Characterization and Engineering 2009;8(2):161-170.

16. Filayson B. Physicochemical aspects of urolithiasis. Kidney Int 1978;13(5):344-360.

17. Mandel N. Crystal-membrane interaction in kidney stone disease. J Am Soc Nephrol 1994;5(5):S37-S45.

18. Selvam R, Ravichandran V. Restoration of tissue antioxidants and prevention of renal stone deposition in vitamin B6 deficient rats fed with vitamin E or methionine. Indian J Exp Biol 1993; 31(11):882-887.

19. Grases F, García-Ferragut L, Costa-Bauzá A. Development of calcium oxalate crystals on urothelium: effect of free radicals. Nephron 1998;78(3):296-301.

20. Kessler T, Jansen B, Hesse A. Effect of blackcurrant, cranberry and plum juice consumption on risk factors associated with kidney stone formation. Eur J Clin Nutr 2002;56(10):1020-1023.

21. Goldfarb DS, Asplin JR. Effect of grapefruit juice on urinary lithogenicity. J Urol 2001;166(1):263-267.

22. Sundaramoorthi P, Kalainathan S. Crystal growth and characterizations studies of SMHP single crystal in silica gel medium and laser induced nucleation reduction process. J Biochem Engg 2007;35(2):244-249.

23. Evan AE, Lingeman JE, Coe FL, Miller NL, Bledsoe SB, Sommer AJ, Williams JC, Youzhi Shao, Worcester EM. Histopathology and surgical anatomy of patients with primary hyperparathyroidism and calcium phosphate stones. Kidney Int 2008;74(2):223-229.

24. Tsay YC. Application of infrared spectroscopy to analysis of urinary calculi. J Urol 1961;86:838-854.

25. Vyas AS, Patel MB, Patel AI, Joshi NR. Evaluation of poly herbal formulation in renal stone disease. Pharma science monitor. Int J Pharm Sci 2012;3(4):2820-2830.

26. Tur JA, Prieto R, Grases F. An animal model to study the effects of diet on risk factors of calcium stone formation. Scand J Urol Nephrol 1991;25(4):311-314.

27. Carbone LD, Bush AJ, Barrow KD, Kang AH. The relationship of sodium intake to calcium and sodium excretion and bone mineral density of the hip in postmenopausal-African-American and Caucasian women. J Bone Miner Metab 2003;21(6):415-420. 